\title{
Intuitive Transformation of UML2 Activities into Fundamental Modeling Concept Petri Nets and Colored Petri Nets
}

\author{
Anthony Spiteri Staines \\ University of Malta \\ Malta
}

\section{Introduction}

\subsection{UML 2 Activity Modeling}

UML 2 activities constitute important modeling notations for specifying and modeling different types of behavior found in systems and object oriented behavior. Activity modeling is useful for understanding and defining web processing, web service composition, business process modeling, workflow modeling, systems integration, task management and low level tasks like software operations. Activity modeling is not just found in UML but also in languages like the systems modeling language (SysML) where activities are modeled using enhanced functional flow block diagrams (EFFBD), business process execution language (BPEL) and Agile. Activity modeling is not restricted to a particular use but can be successfully integrated into different approaches as required.

UML 2 activities are classified into i) fundamental, ii) basic , iii) intermediate, iv) complete, v) structured, vi) complete structured and vii) extra structured. Each class is useful for a particular problem area. E.g. structured activities address traditional programming language modeling, whilst fundamental and basic activities are ideal for high level business process modelling (Spiteri Staines, 2008).

\subsection{Petri Nets and Activity Semantics}

Activities are based on Petri Net like semantics, existing at a higher level of abstraction. They share common properties with Petri nets, although these are loosely defined in the UML 2 Superstructure specification (OMG, 2009). Rules for node execution and tokens are presented. There are also complex rules for token flows. New extensions, new rules and complex token types are mentioned. Unfortunately, the rules for activity execution are not clearly explained and defined in the UML specification. Problems can arise for concurrency, synchronization and other types of behavior. UML activities lack proper formal concepts. Activities are unsuitable for simulation, and analysis, lacking proper validation and verification techniques. The activity diagram constructed is a non executable model.

Petri nets are formalisms for describing or explaining the partial ordering of system events or actions, following a particular temporal order. In a Petri net event sequencing depends on 
the system state following specific rules. Knowing the causal event ordering and current state gives predictable behavior. Petri nets seem to be the best natural choice to support activities for various reasons. Evidence in the UML Superstructure specification suggests activities to be derived in part from Petri nets. Petri net formalisms can support all activity constructs and more complex modeling. They have over three decades of coverage, and are based on sound mathematical properties. Petri nets are useful for visualization, formal verification, simulation and detailed analysis. Transforming UML 2 into Petri nets creates some new issues: i) which classes of Petri Nets are best suited for conversion, ii) how to convert, iii) visualization issues. The best classes to support activity modeling are higher order net and Colored Petri net (CPN) classes. However if activities are directly translated into CPNs, these will normally contain more nodes and edges than the actual activity diagram. One solution is to initially transform the activity into a Fundamental Modeling Concept Petri Net diagram concise notation and later construct the detailed CPN.

UML 2.2 Activity diagrams (OMG, 2009) introduce many new concepts and notations not present in UML 1.x. Some of these are collections, streams, loops and exception handling mechanisms. UML 2.2 replaces UML 1.5 'Activity graph' concepts based on state machines with activity modeling that is supposedly based on Petri net semantics (Borger et al., 2000). This is because the 'Petri net' like semantics offer advantages such as the possibility of multiple and parallel flow modelling, better control and sequencing, etc. The UML super structure specification (OMG, 2009) gives basic rules for node execution and tokens. Tokens are removed from nodes and offered to output edges. UML rules for executing an action are surprisingly similar to Petri nets, although the definition is not clear. New extensions may add new token types having their own flow rules making things more complex.

The following explain some main properties of activity diagrams:-

i) Activity diagram nodes have flow-of-control constructs like synchronization, decision, concurrency and sequence. These are fundamentally similar to those of Petri Nets.

ii) Activity diagram semantics are based on token flows (OMG, 2009). Tokens can contain objects, data, control information. Tokens are normally distinguishable through an individual time-stamp.

iii) These classes include task sequencing, data flows and control flows based on normal resources. The activity types suited to convert into Petri net models are fundamental, basic and intermediate, although conversion work can be extended to other classes.

This work will explain how UML activities can be easily transformed or converted into Petri nets, FMC-PND and CPNs. A case study of a web ordering system is given to illustrate this approach.

\section{Transformational Mapping of Activities into FMC-PNDs and CPNs}

\subsection{Related Work}

This section presents some evidence of Petri Net use for supporting UML activities which is based on available literature. Basically there are two mainstream approaches to transform UML constructs into Petri nets. The approaches are i) informal and ii) formal. Not all approaches use higher order nets, so some information loss might occur.

In (Canevet et al., 2004) a UML 2 activity model for an online multi role playing game is transformed into a PEPA net and analyzed. There is no explanation of the translation process, which seems to be informal. This indicates the usefulness of CPNs for requirements 
engineering. Another method suggests mapping of UML use case constructs to CPNs based on multi layers (Shin et al., 2003). UML use cases are the starting point for activity modeling. No proper formalisms have been used. Statecharts are easily converted into OPNs (Saldhana \& Shatz, 2000). Basic constructs have been mapped informally to CPNs for consistent models (King \& Pooley, 2002; Shinkawa, 2006). CPNs have been successfully applied in the modeling of a real bank's advisor portal system's use cases. A complete system was constructed and implemented based on CPNs (Jørgensen \& Lassen, 2006).

In (Lopez-Grao \& Campos, 2004; Merseguer et al., 2006) a well structured, semi formal method is presented to translate activities into LGSPNs (labeled generalized stochastic Petri nets). These are very useful for performance analysis but the final net is a simplification of the activity diagram and includes the time dimension. LGSPNs cannot contain all the details CPNs can have, although they are very useful for performance estimation. The LaQuSo project (LaQuSo, 2007) has case tools to transform activity diagrams into simple Petri nets for soundness analysis.

UML can be formalized using Petri net like semantics. HLTPNs (higher level timed Petri nets) which are similar to CPNs (Baresi \& Pezze, 2001) suggest this. In (Garrido \& Gea, 2002) a CPN based formalization of the UML is presented. In (Störrle, 2004a) a simple formal transformation is presented, this idea is explained in section 4.2. Looking up literature other various examples and approaches are possible.

FMC-PND are based on the concepts found in traditional Petri nets and higher order Petri nets. Fundamental modeling concepts, as the name implies focus on practical and effectively understanding structures in the real world. FMC diagrams have been successfully applied to model various ERP projects at SAP, client server activity and even basic algorithms (Knöpfel et al., 2006). These diagrams are based on visualization concepts and pattern identification. This modelling approach is easy to apply for systems and software design. Special tools are not required.

\subsection{Reasons for Mapping and Transforming Activities into Colored Petri Nets}

Several motivating factors exists for mapping UML activity diagrams into Petri nets (Baresi \& Pezze, 2001; Bock, 2005; Canevet et al., 2004; Dumas \& Ter Hofstead, 2001; Ghodsi \& Kent, 1991; Hu \& Shatz, 2004; Jancar et al., 1999; Lopez-Grao \& Campos, 2004; Petriu \& Shen, 2002; Sivaraman \& Kamath, 2003; Störrle, 2004a,b; Van der Aalst, 1998; Ziaei \& Agha, 2003). i) UML lacks support for strong simulation and analysis techniques (Baresi \& Pezze, 2001), ii) According to the OMG, UML 2 activity diagrams are based on Petri net semantics making them the best choice, iii) Petri Nets are graphical formalisms, iv) Petri nets model all UML activity constructs, v) Petri nets can be validated, verified and simulated, vi) Petri nets have sound mathematical properties, vii) Petri nets are suitable for visualization, aesthetic representation of systems, etc. (Knöpfel et al., 2006, Kristensen et al., 1998, 2004; Van Hee, 2005).

UML activities can also be transformed into other formalisms such as process algebras, formal languages, logics, CCS, etc. In (Störrle \& Hausmann, 2005) it is explained that intuitive mapping from UML 2 activities into other formal notations such as algebras, CCS or other transition systems does not exist. There is considerable separation between the actual syntax and semantics. Most formalisms are non graphical and do not support visualization. 
Higher order Petri nets (Jancar et al., 1999; Störrle, 2004; Störrle \& Hausmann, 2005) are best suited to activity modeling. Activities or actions are defined using token types, objects, sets, colored sets and advanced data types. Combining arc inscriptions with transitions and special languages expand the modeling capabilities. Colored Petri Nets (CPNs) (CPNTools ,2009; Jørgensen ,2002;Kristensen et al. ,1998; Kristensen, 2004) based on languages like ML are good for activity modeling. The ML and CPN ML languages can completely imitate the behavior of activity diagrams ranging from streaming / non streaming activities, multiple control flows, control buffers and pins, timing issues, etc. Some of these are part of the SysML language (Bock,2005). CPNs show a close correspondence to activities and offer enhanced modeling even though other Petri net classes are still useful.

However problems are created when transforming complex activity diagrams directly into CPNs and other high level Petri net classes as is evidenced in (Dumas \& Ter Hofstead, 2001; Hu \& Shatz, 2004; Jancar et al, 1999; Petriu \& Shen, 2002; Sivaraman \& Kamath,2003; Störrle, 2004 a,b; Störrle \& Hausmann, 2005; Van der Aalst,1998). Some are listed below:

- Constructing the $\mathrm{CPN}$ is a long and tedious task requiring expert knowledge.

- The CPN might end up have substantially more nodes and edges than the UML diagram. This is the case if a one-to-one mapping between the activity diagram and the CPN is maintained. Additional places need to be added for concurrency and other activities.

- Visually the constructed CPN might be difficult to read and understand, i.e. it is unsuitable for visualization.

- Other constructs like sets, data types, functions, etc. must be defined and programmed to make the CPN operational. These are not available directly from the activity diagram.

It is important to find solutions for these issues. CPNs are the best suited Petri net class for modeling activity diagrams. Unfortunately they can become complicated for stakeholders to comprehend (Dori,2003; Kaindl \& Carroll,1999; Soderborg et al., 2003). A solution should simplify the comprehension whilst retaining the fundamental features. Models must be clear, simple and precise for proper comprehension by stakeholders during the analysis phase.

\subsection{A Fundamental Modeling Concept Petri Net and Colored Petri Net Solution}

An intuitive mapping or transformation of UML activities into Petri nets and vice-versa is possible. This is because UML 2 activities are based on Petri net concepts and add other details.

The fundamental modeling concept (FMC) idea (Knöpfel et al.,2006) originated at the HPI Institute, Potsdam, Germany. FMC present visualization guidelines to creating models that are more comprehensible, compact and easier to construct. The idea in this work is to apply the principles of FMC to construct a comprehensible Petri net or CPN model from the UML activity. To this end reduced FMC-PND are suggested. FMC-PND can serve to represent repeated patterns with emphasis on the layout of the notational elements clearly explaining what is happening.

The proposed solution is to i) identify the activity diagram, ii) transform it into Fundamental Modeling Concept Petri net diagram (FMC-PND) and iii) construct a CPN. Steps ii) and iii) can be carried out concurrently. 
FMC focus on system related structures, system behavior at a high level and performance. UML 2 activity diagrams focus on a wide spectrum of issues complicating things. UML activities are more suited to model software behavior. FMC-PND (Knöpfel et al.,2006) offer several advantages over creating an immediate CPN simplifying the UML activity diagram. A FMC-PND (Knöpfel et al.,2006) is readable and simpler to create than a CPN. At this point, understanding the system and identifying patterns are more important than execution.

- $\quad$ FMC are based on i) abstraction, ii) simplicity, iii) universality, iv) separation of concerns, v) aesthetics and secondary notation.

- $\quad$ FMC are visually attractive.

- $\quad$ FMC are based on specific principles like diagram size, node arrangement, node shape, enumeration, harmonization, and proper labeling making the diagrams easier to comprehend.

- $\quad$ FMC are based on pattern identification. Repeated patterns can be simplified or reduced.

- $\quad$ FMC present a way to abstract process representation and to study the activities involved and reengineer the system.

- The complete work behind FMC is documented in (Knöpfel et al.,2006). FMC model systems on the static and dynamic aspect, they are based on proven techniques and have been used to design interactive software systems (Gröne et al., 2003, 2004, 2006; Tabeling, 2002, 2004; Tabeling \& Gröne, 2005).

The FMC-PND is just a block diagram or visual representation. The aesthetical layout of the model places strict emphasis on the layout of notational elements, nodes and edges to present better graphical structures and patterns. These are suitable for comprehension by different system stakeholders. On the other hand for proper execution, analysis and performance estimation, the activity should be converted to a CPN.

\section{Transformation Approach}

\subsection{Activities and Corresponding Petri Net Notations}

Referring to previous work presented in (Störrle, 2004 a,b; Störrle \& Hausmann, 2005), the activity diagram specification in (OMG, 2009) and working experience with Petri nets, it is possible to find and define combinations for converting or transforming activity semantics into their Petri net counterpart.

Normal activity edges are converted into Petri net places with input and output arcs. There are exceptions when the activity edge links: i) merge node to merge node, ii) start node to merge node, iii) merge node to activity final node, iv) merge node to flow final node. These convert to a Petri net transition with input and output arcs. Other activity edge exceptions exist, i) edges outgoing from a start node, ii) activity final incoming edge and iii) flow final incoming edge, here these convert into a Petri net arc or flow. Conversion for object nodes and signal nodes are included in a separate table. 


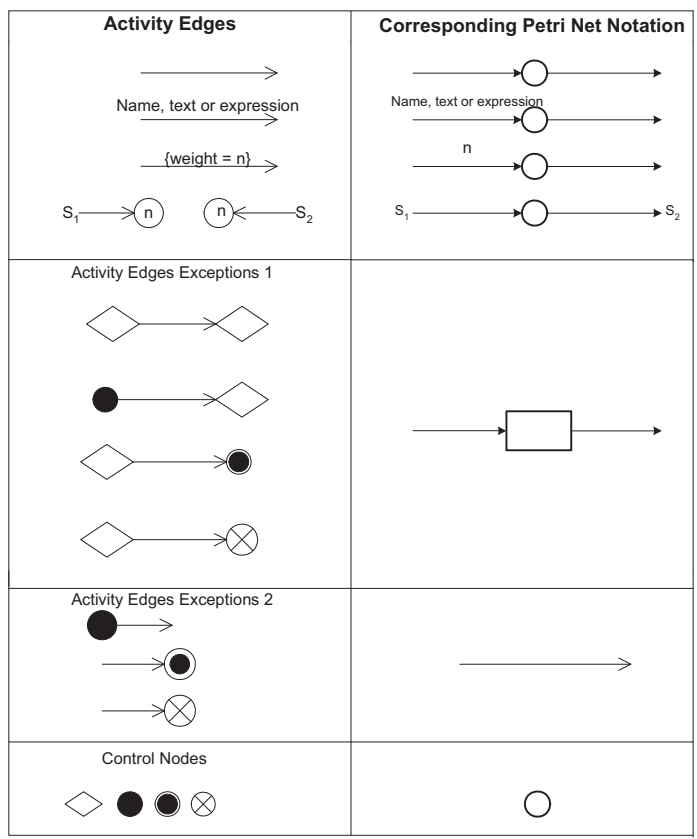

Table 1. Activity Edges and Control Nodes corresponding Petri Net notations

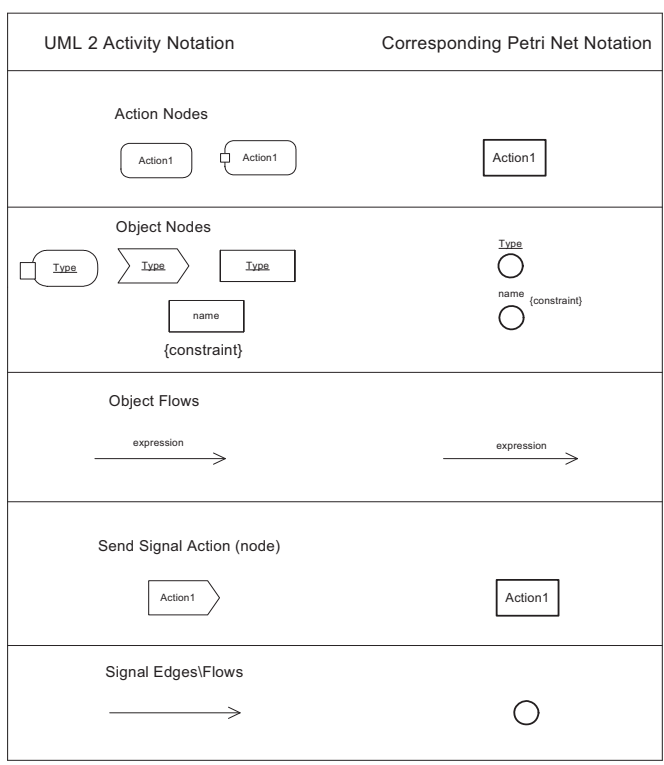

Table 2. Other Activity Notations with corresponding Petri Net notations 
The notations shown in Table 1 and 2 summarize the main notations that are used in UML 2 activity models. Table 1 and 2 explain this conversion process taking into consideration many of the constructs found in UML 2 activities, although there are exceptions. From table 2 it is evident that the conversion is similar to that of normal activities and edges except for object flows. Normally activity diagrams would be made up using the main notations.

The UML superstructure specification defines a variety of presentation options that can be used in conjunction with these notations. The notations and their depiction vary, depending on the type of activity that is being modeled. It is possible to annotate these notations to show more detail. Changing the shape or way a notation is depicted will have no effect on the corresponding Petri net.

Table 3 shows signal node exceptions. There is an accept signal, with no incoming edges. This type of signal is always enabled, hence it initiates an activity or series of actions. The other type of signal node is a repetitive time event. It means that this event occurs at regular time intervals. In these cases a Petri net token generator fits closely with this behavior. Alternatively, a single place, with tokens always available can be used.

\begin{tabular}{|c|}
\hline Signal Node Exceptions \\
Accept Signal \\
(no incoming edges) \\
always enabled
\end{tabular}

Table 3. Signal Node Exceptions and Corresponding Petri Net

\subsection{Conversion of Enhanced Activity Structures}

An expansion region is convertible into a transition. The transition can be decomposed further to sub levels.

i. Object node pins are just an alternative style for display object nodes. Hence they do not affect the conversion process.

ii. Parameter sets are used to direct token flows. Petri net places can be used for stand alone pins.

iii. Parameter nodes are similar to object nodes. They have no significant effect on the resultant Petri net. They are useful for partitioning, thus places could be used for parameter nodes, just as is done for object nodes. 

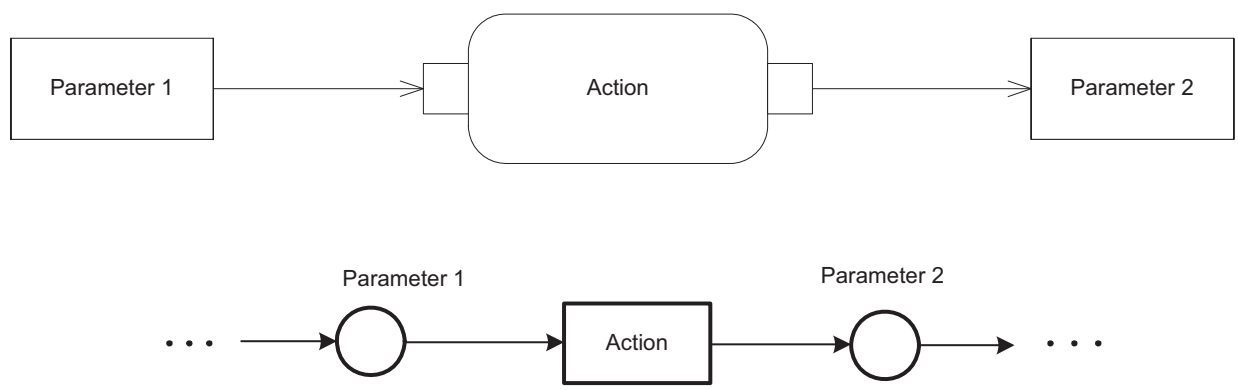

Fig. 1. Activity with Parameter Nodes and Corresponding Petri Net

iv. Partitioning and swim lanes of the activity model have no effect whatsoever on the Petri net model.

v. For intermediate activities, special types of nodes are possible. E.g. $<<$ central buffer $>>,<<$ data store $>>$. Some of these can be represented using a single place. They can be decomposed to a full Petri net model with detailed behavior .

\subsection{FMC-PND Basic Patterns}

When examining activity models, four or five basic patterns well known to workflow nets and BPEL (Van der Aalst, 1998), generalized task graphs (GTG) (Ghodsi \& Kent, 1991), task graph mappings (Chen et al., 1995) and FMC can easily be identified.

The patterns are classified as: i) sequence, ii) and/split join, iii) xor split, ,iv) xor join and v) iteration. Having FMC principles of abstraction, simplicity, universality, separation of concerns, aesthetics and secondary notations (Knöpfel et al., 2006) a simplified, neater and comprehensible Petri net can be drawn.

Fig. 2-6 illustrate from left to right the: i) activity diagram pattern, ii) Petri net and iii) reduced FMC-PND. From previous work presented in (Störrle, 2004 a,b; Störrle \& Hausmann,2005) the activity diagram specification and practical experience with Petri nets, combinations to convert or transform activity semantics into their Petri net counterpart are easily discovered.
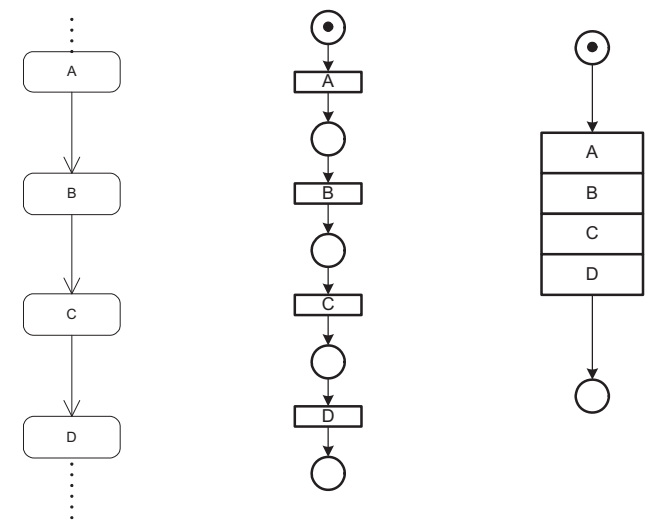

Fig. 2. Activity Sequence 

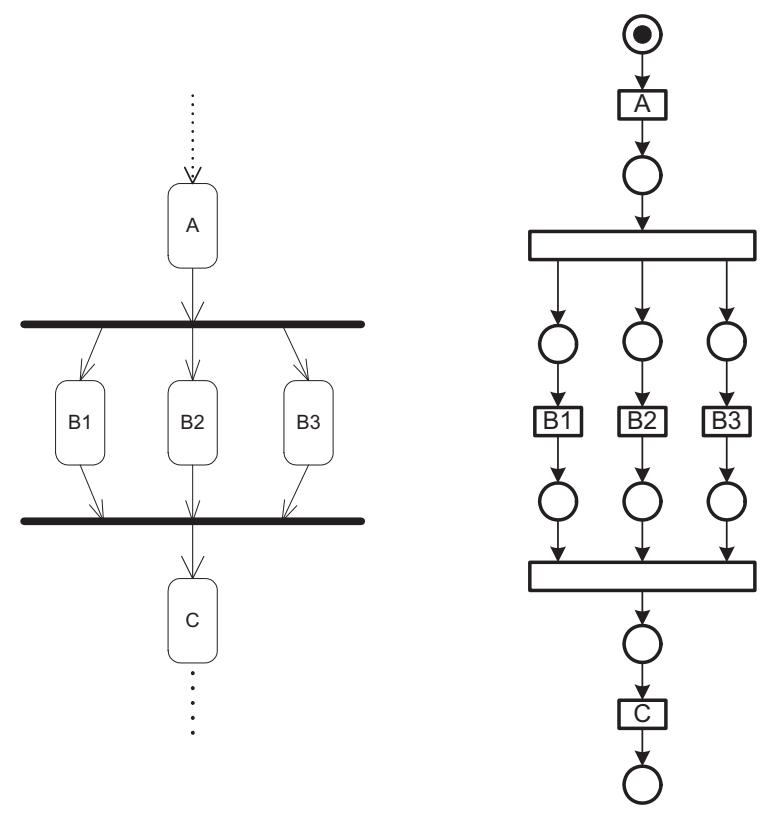

Fig. 3. Activity Fork and Join
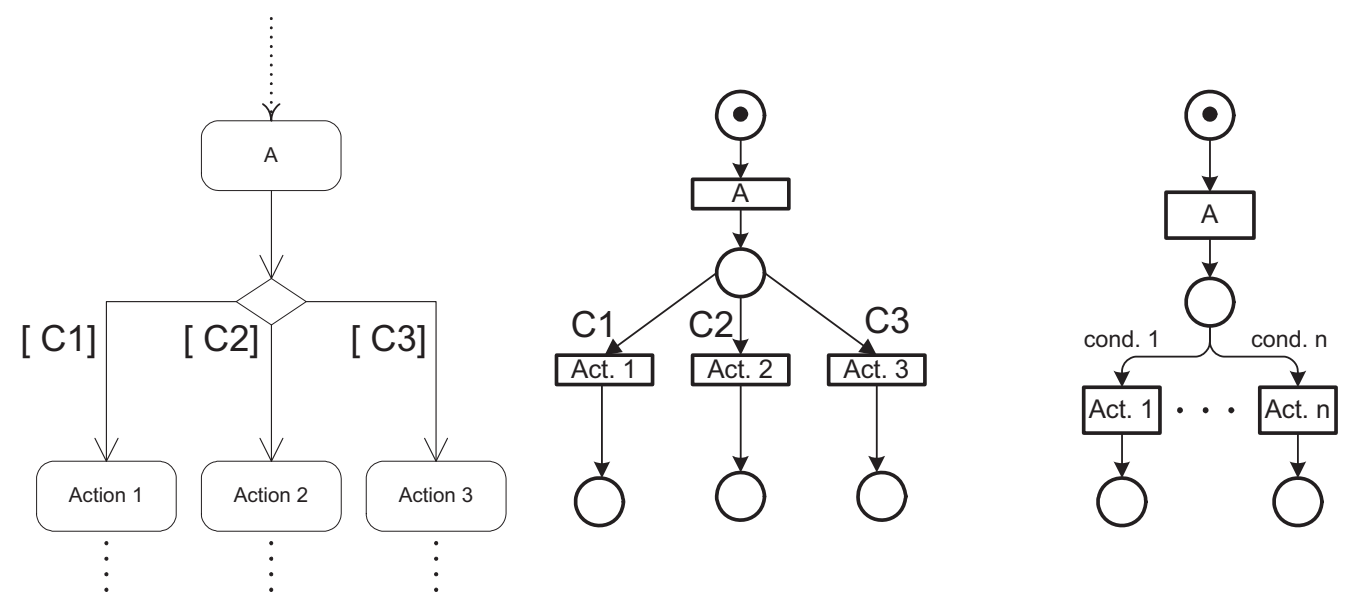

Fig. 4. Activity Decision/Choice Node 

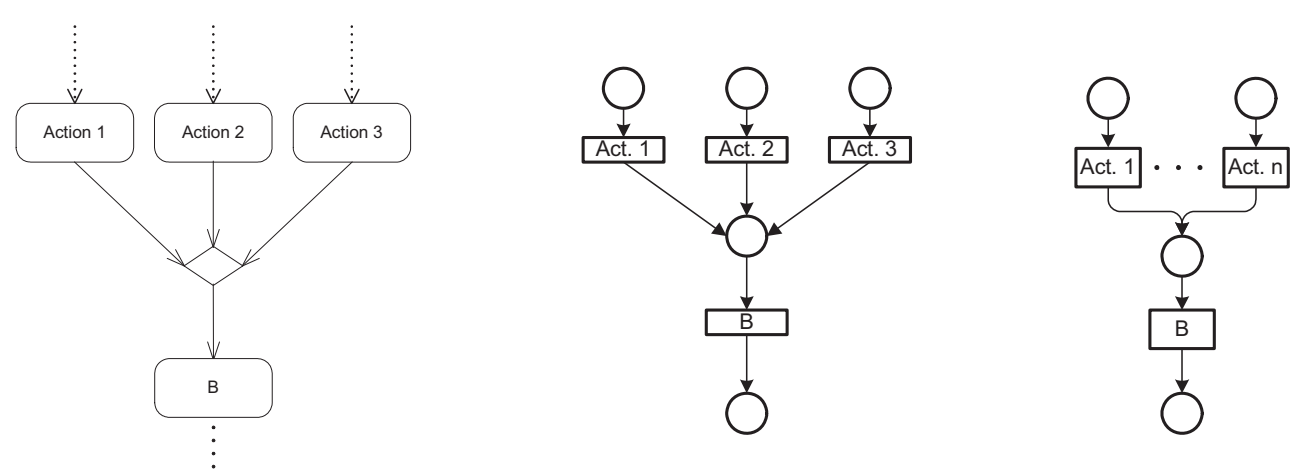

Fig. 5. Activity Merge Node
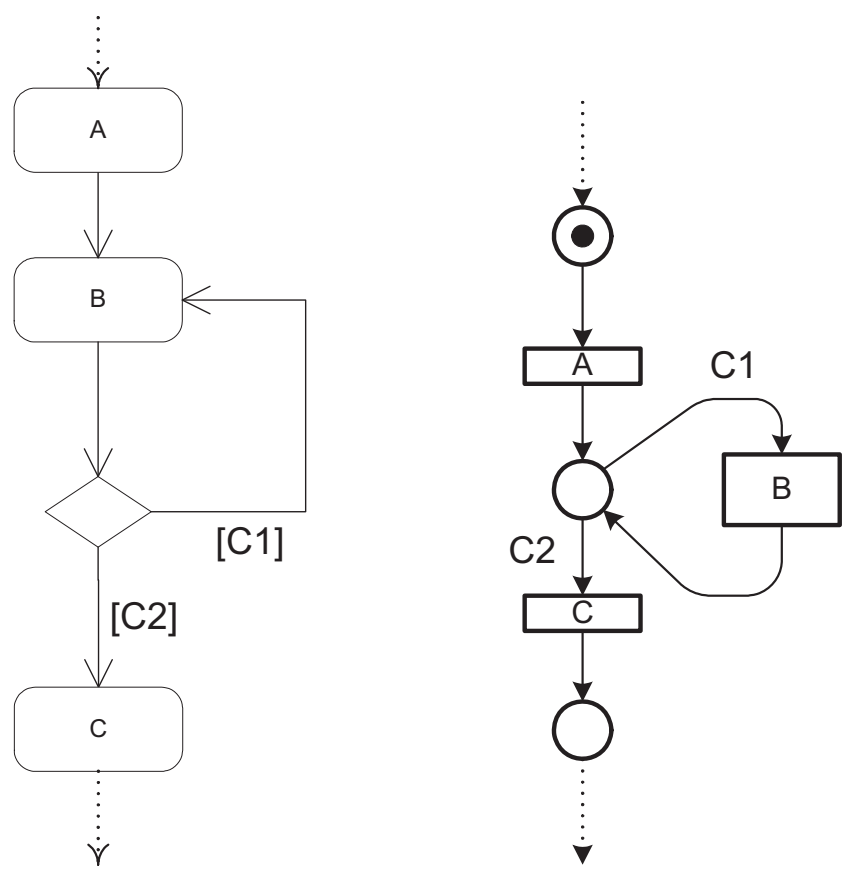

Fig. 6. Activity Iteration

The UML 2 activity semantics cater for special cases. Advanced constructs like buffers, client server communication, etc. can be used. These can also be modelled using FMC patterns. Fig. 7 shows an exception handler not found in UML 1.x. The complete Petri net for the exception handler is quite long. The reduced FMC Petri net simplifies this, showing some similarity to the activity diagram. Swimlanes, expansion regions, pin notations, parameter sets, interruptible regions similar to exception handling in fig. 7 can be modeled with FMC and CPNs. 
Intuitive Transformation of UML2 Activities

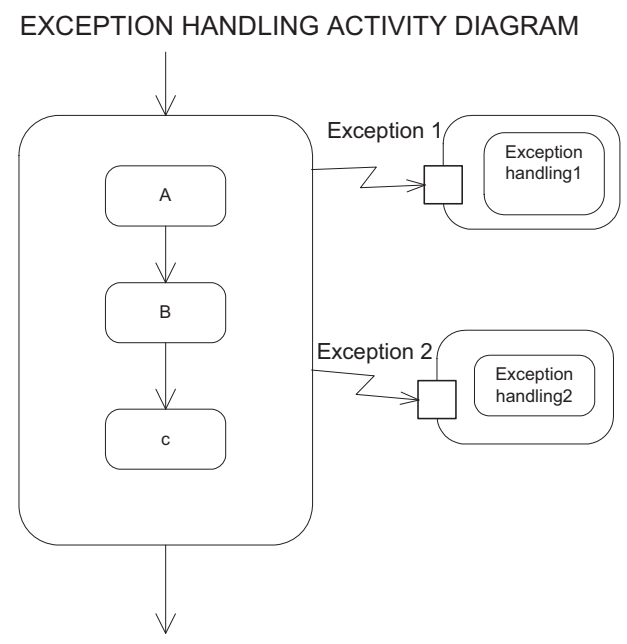

\section{EXCEPTION HANDLING FMC PETRI NET}
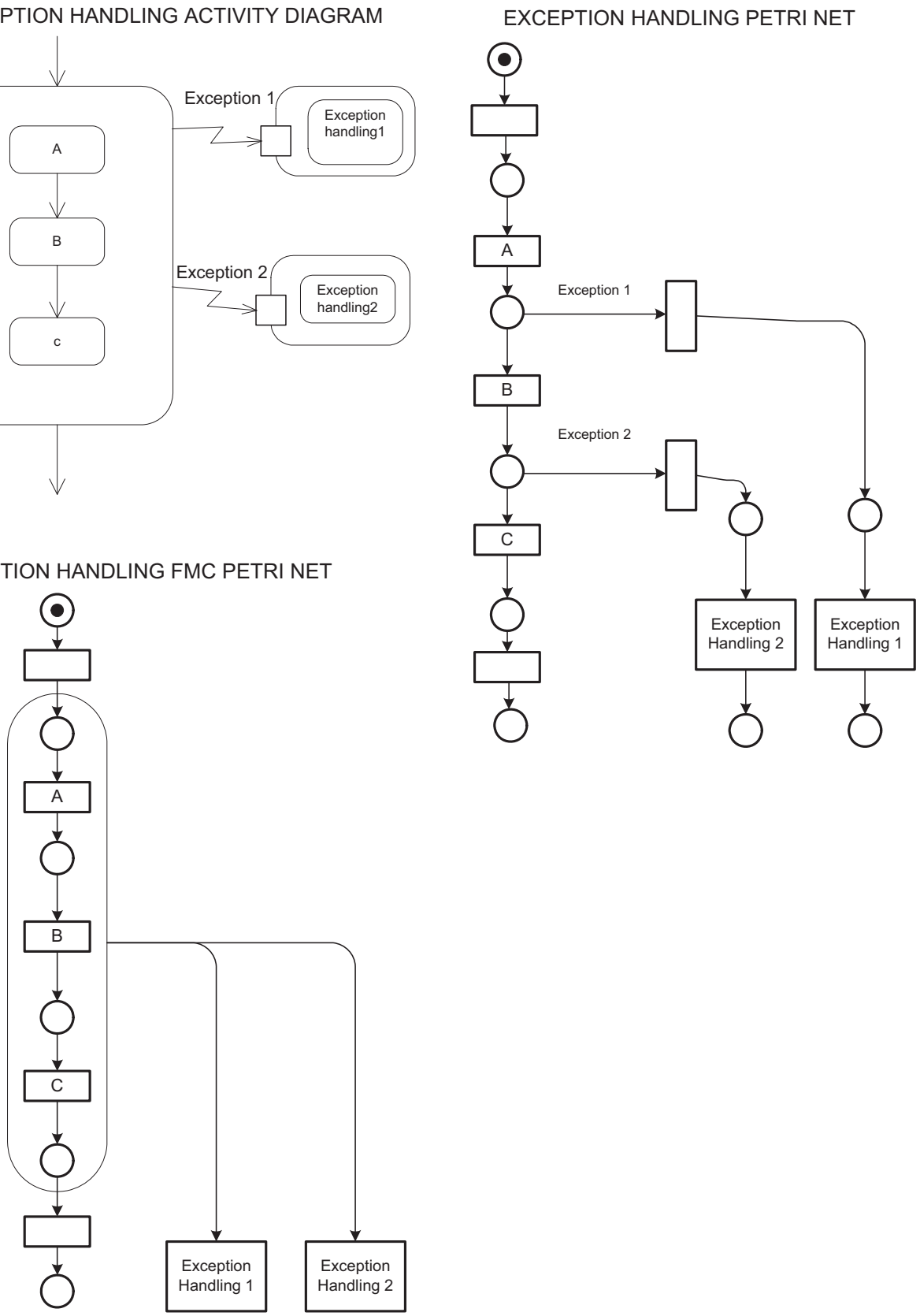

Fig. 7. Exception Handling 


\section{Transformational Approach for Activity to Colored Petri Net}

\subsection{Simple Conversion}

Conversion from the activity model to the CPN can be done simply by looking up tables 1-3. The algorithm outline proposed for this is:

If normal (edge or node) lookup corresponding petri net notation and replace

Else lookup exception and replace

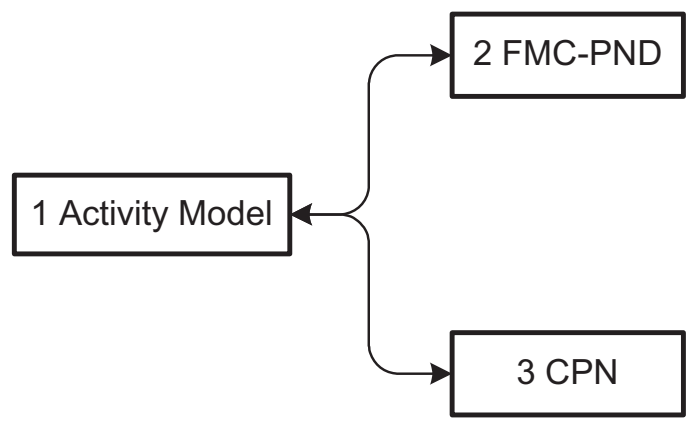

Fig. 8. Model Mapping Informal or Formal

Fig. 8 shows how the activity model could be used for the FMC-PND and the CPN. The arrows connecting the models are bi-directional indicating that a change to one of the models must be reflected in the others. The FMC-PND is of greater interest to the shareholders, it is concise and more readable. On the other hand the CPN has more detail. It is suitable for execution and validation. The FMC-PND focuses on activities, ignoring boxes, input pins and other UML constructs, etc. The main patterns listed in 3.3 should be identified and the resultant Petri net is simplified. Using FMC principles the Petri net is more readable and easy to visualize.

\subsection{Formal Mapping and Transformation}

To obtain a fully functional CPN model, the input and output arcs have to be defined from an existing color set. Places must have corresponding color. Normally this information is not available from the UML activity. E.g. Places have type definitions. Arcs might be required to have functions. This information is not available from the activity which is normally a non executable model. The arc expression function, the initialization function, etc. are not directly obtainable. The transformation of UML 2 activities into a CPN has been formalized (Störrle, 2004b, Garrido \& Gea, 2002). In (Störrle, 2004b) a semantic function is to used map activity nodes or edges into a CPN. The function converts an activity diagram <activity node, activity edge $>$ into a CPN defined as $<\mathrm{N}$, SigAlg, color, guard, effect $>$, where $\mathrm{N}=<\mathrm{P}, \mathrm{T}, \mathrm{A}>$. A set of transformation mappings for UML into CPN can be defined. The FMCPND are based on templates. The CPNtool (CPNTools, 2009) uses XML format.

The CPN can be defined as nine tuple set $=\left(\sum, P, T, A, N, C, G, E, I\right)$, where $\sum$ is a finite set of non-empty types or color sets. $\mathrm{P}$ is a finite set of places. $\mathrm{T}$ is a finite set of transitions. $\mathrm{A}$ is a finite set of arcs. $\mathrm{N}$ is the node function. $\mathrm{C}$ is a color function. $\mathrm{G}$ is a guard function. $\mathrm{E}$ is an arc expression function. I is an initialization function. The information below explains in 
simple terms the transformation of activity constructs into CPN constructs. The activity constructs can be stored in a list having <activity nodes, activity edges>. The information below can be used to construct a semantic function for the transformation.

i. Initial node, final node, object nodes, control nodes, signal edges and activity edges excluding exceptions all transform or correspond to places

ii. Action nodes, control nodes, signal nodes, activity edge exceptions1 transform or correspond to transitions

iii. Object flows, activity edge exceptions 2, activity edges transform or correspond to arcs connecting to input flows and output flows

iv. Object node types, object types, control node types, parameters, etc. correspond with the color type of the CPN places

v. Expressions on activity edges, object flows correspond to expressions or arc inscriptions on the corresponding CPN arcs.

vi. Guards at merge, fork or joining nodes convert to expressions on the corresponding arcs based on the arc inscription language.

\subsection{Triple Graph Grammer Correspondence}

The activity diagram data could be mapped directly into a CPN using a model-to-model mapping approach based on the OMG QVT (Query View Transform) or ATL (Atlas Transformation Language). According to (Lohmann et al., 2007) Triple Graph Grammars (TGGs) are better to express workflow patterns of UML activities as we have graph-to-graph mappings. TGGs seem to offer the best choice for mapping activity models into CPNs and FMC-PNDs. The concepts behind the QVT of the OMG standard are surprisingly similar to TGG. The idea behind TGG is to keep a transformational correspondence between two different models (Kindler \& Wagner, 2007).

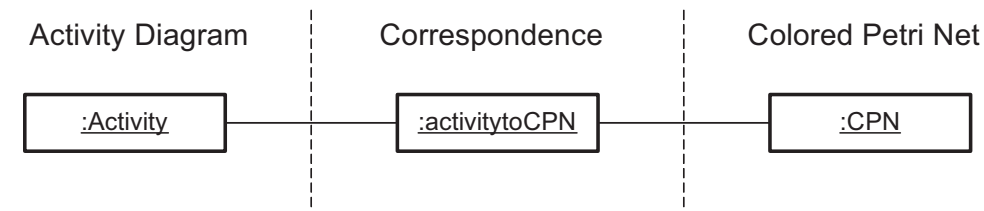

Fig. 9. Triple Graph Grammar High level Correspondence between Activity and CPN

Figure 9 shows high level correspondence between the activity model and a CPN using TGGs. The correspondence will define all the transformations or mapping rules from the activity into the $\mathrm{CPN}$ and vice-versa. These rules can be built using the information given previously. New rules can be added to ensure that the Petri net is complete, etc. Rules for reduction for identifiable patterns can be included.

The transformations can be forward or backward. E.g. if changes are done to the activity these are automatically reflected in the CPN. The opposite is also true, i.e. certain changes to the CPN result in automatic updates in the activity model. This approach can even include correspondence with the FMC-PND. Literature is available about TGGs in some publications. 


\section{Case Study: An Activity Model for Online Ordering}

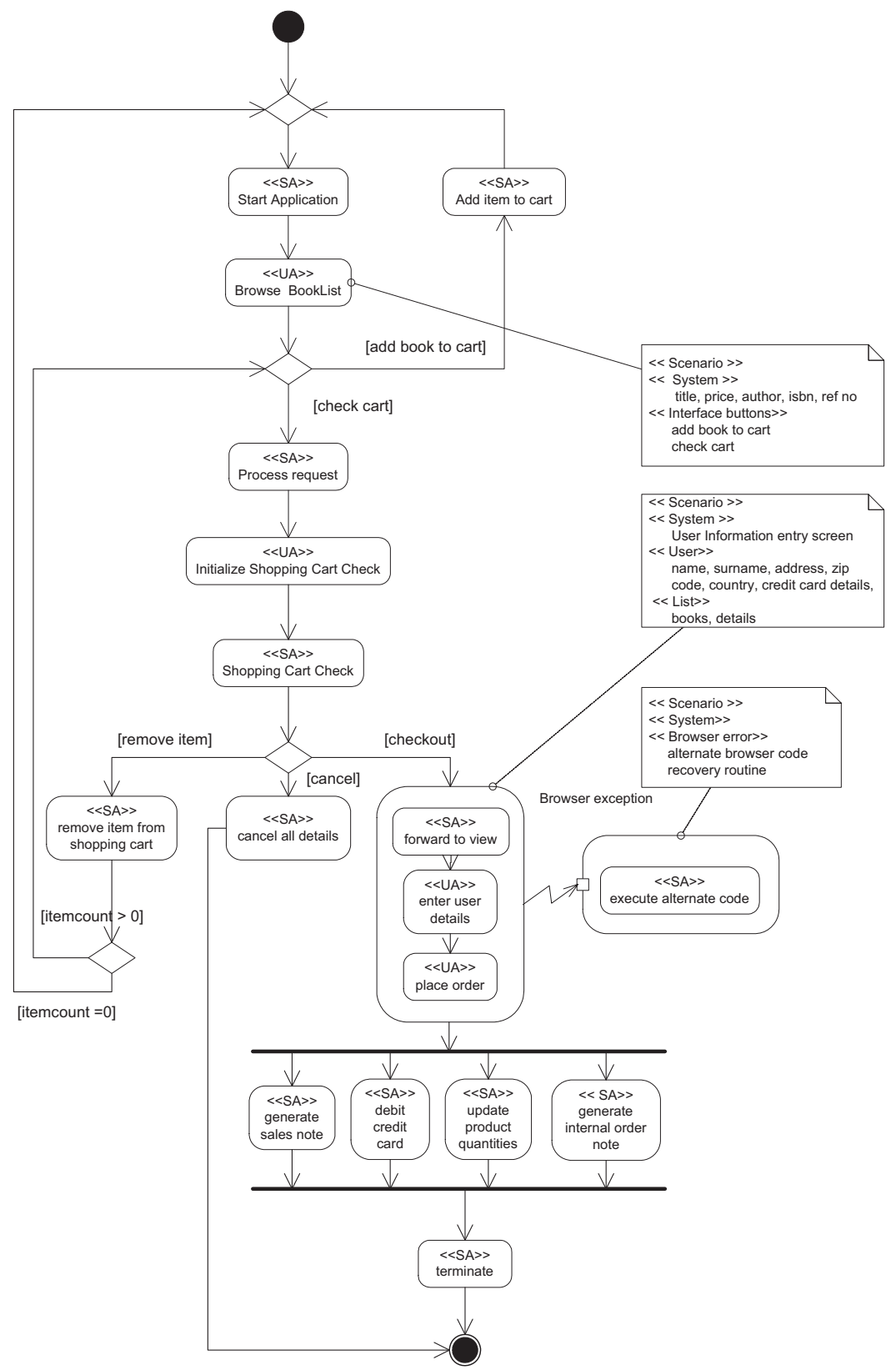

Fig. 10. Activity for An Online Book Ordering System 
An activity model of an online book ordering system is shown in fig. 10. The diagram is based on an approach in (Lorenz \& Six, 2006) where user oriented and interaction oriented activities are combined into a specification oriented diagram. This activity can be mapped to a target platform like J2EE with web tier, struts, BPEL, etc., implementing the programming code directly.

The activity model combines three different views. These are i) user oriented, ii) interaction oriented and iii) specification oriented. The specification oriented view is the final result. Most of the activities are "system activities" and hence stereotyped $<<$ SA $>>$ along with "user activities" stereotyped $<<\mathrm{UA}>>$. Ordering involves adding books to a cart. A user can include new books. At the end the user can i) cancel, ii) remove books and continue adding or iii) checkout. Checkout then proceeds to placing an order where the name, address, credit card details, etc. are required. Then a sales note, internal note and debit note are generated. The product quantities are updated. An exception handler checks for browser errors. When an error occurs alternative code is executed. This activity includes three possible iterations. There are two termination options either i) cancel or ii) checkout. Notes are added for better comprehension in this example. The diagram is becoming more complex to interpret. For non-technical stakeholders there is a comprehension problem.

\subsection{Fundamental Modeling Concept- Petri Net for Online Ordering}

Using the activity in fig.10 and the information given in section 3.3 it is possible to create the FMC-PND. The model follows the FMC principles listed in 2.3. These are explained in (Knöpfel et al., 2006).

Compact representation can be used for sequences, error alternatives, choice and merge. A simpler model which is easier to read is produced. This can be seen from the diagram shown in fig. 11. Interpretation is simpler. The important processes for ordering are clearly highlighted. The activity model combines three different views. These are i) user oriented, ii) interaction oriented and iii) specification oriented. The specification oriented view is the final result. Most of the activities are "system activities" and hence stereotyped $<<$ SA $>>$ along with "user activities".

\subsection{Colored Petri Net for Online Ordering}

If the activity model is structurally correct it is possible to construct the CPN. The CPN is similar to the FMC-PND but contains more places and transitions. These have to be added to model precisely the UML 2 activity in fig. 10. Additionally the CPN construction is necessary to build an executable model for testing and verification. The most adequate tools to construct the CPN are CPNTools (Kristensen et al. 1998, CPNTools, 2009).

The final CPN is shown in fig. 12. This shows the online book ordering activity. It is a fully working model containing detailed processing logic, bringing the model as close as possible to the real scenario. The complete executable specification was created for the book ordering system. The payment and credit card details can be entered as a string. Random generators are used to generate random book order numbers; price and random errors are generated as required. The programming needed for the model was done using CPN ML. The functions used are listed in fig. 12. Extensive code that has not been presented here was needed for perfect execution. If problems turn up during execution these are seen visually and they can be immediately amended in all the models. 


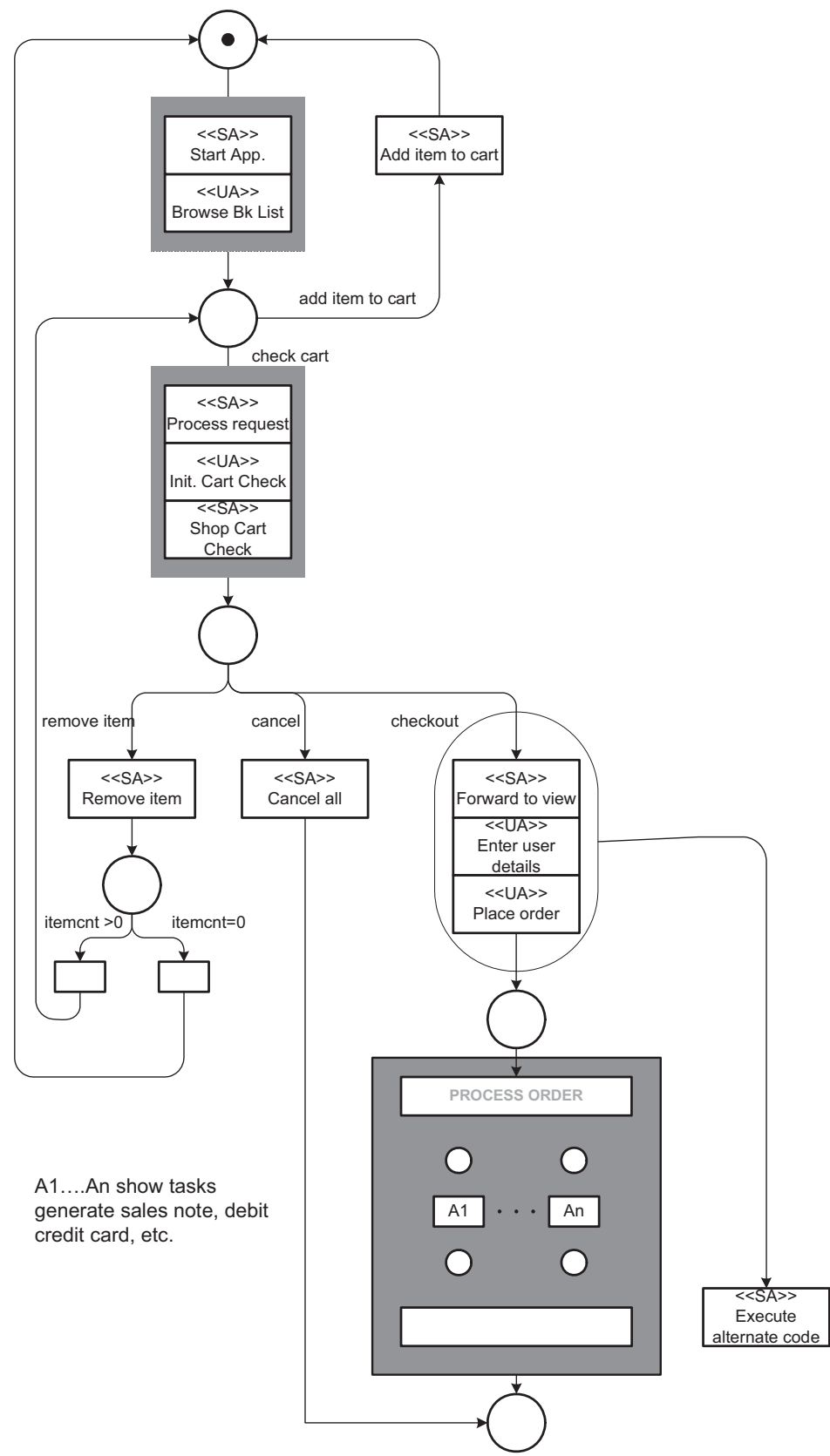

Fig. 11. Fundamental Modeling Concept Petri Net For Online Book Ordering 
Intuitive Transformation of UML2 Activities

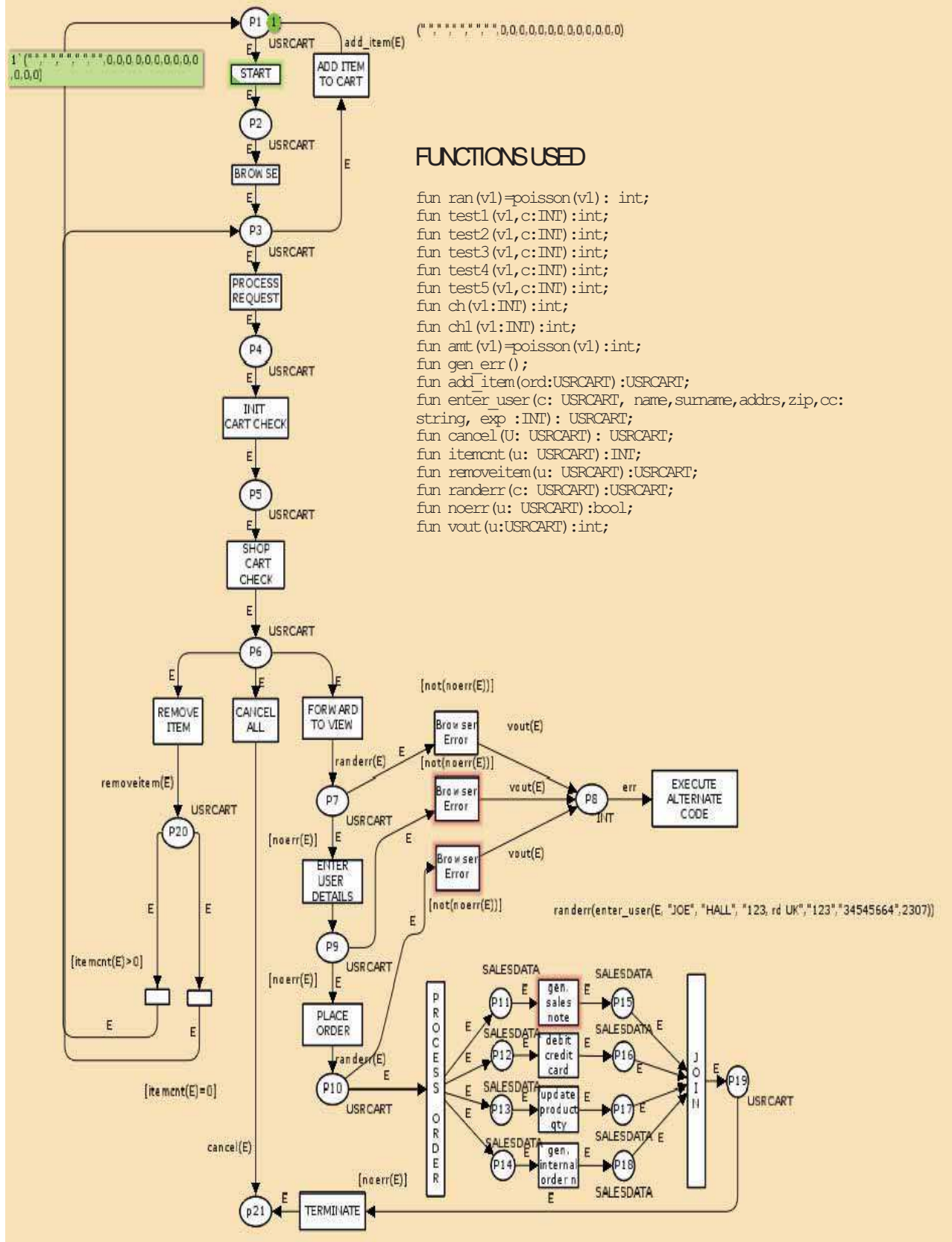

Fig. 12. Colored Petri Net For Online Book Ordering 


\section{Brief Analysis of the Modeling Approaches}

In activity modeling, processes should have a clear start and controlled termination (Van Hee, 1995,2005). UML activities, model processes as abstract entities. These are converted into well defined executable processes in the CPN. The FMC-PND helps to properly comprehend the CPN model. The CPN is validated and tested using the CPNTools. Once the models are finalized they can be used to define an executable specification.

Activity modeling covers a variety of system aspects. Three possible checks that can be carried out to a system (Van Hee, 2005) are identifiable. These are i) reviews, ii) proofs and iii) tests. The activity diagram and the FMC-PND are suitable for i). The CPN is suitable for ii) and iii). The FMC-PND can be used for conformance analysis based on balanced and /or splits or joins, conflict issues, well structured and well handled nets principles (Van der Aalst, 1998). The CPN is suited for performance analysis and can be analyzed using classic Petri net theory like linear algebra, place invariants, transition invariants, non reachable conditions, soundness, reachability tree, model checking, reduction techniques, markov chain analysis, etc. It can also be checked for deadlock and livelock thus eliminating logically incorrect specifications.

The FMC-PND is definitely more simple, understandable and readable than the CPN or other higher level nets, having just 9 places (see fig. 11) compared to the 21 places in the CPN (see fig. 12). This implies that there are substantially less nodes and edges in the FMCPND. The resultant diagrams in fig. 11 and 12 are cyclical and not choice free, i.e. there are some conflicting transitions. Some parts of the net are strongly connected and other parts loosely connected. The patterns presented in the FMC-PND can be used to reduce the complexity in the activity diagram.

\begin{tabular}{lllll}
\hline $\begin{array}{l}\text { Model } \\
\text { Type }\end{array}$ & $\begin{array}{l}\text { Pattern } \\
\text { Based }\end{array}$ & $\begin{array}{l}\text { Suitability for } \\
\text { Visualization }\end{array}$ & $\begin{array}{l}\text { Suitability for } \\
\text { Execution }\end{array}$ & $\begin{array}{l}\text { Testing and } \\
\text { Verification }\end{array}$ \\
\hline UML ACTIVITY & No & Moderate & n/a & possible \\
FMC-PND & Yes & Very good & n/a & possible \\
CPN & No & Moderate & YES & possible \\
\hline
\end{tabular}

Table 4. Common Properties for: UML Activity, FMC-PND and CPN

Table 4 indicates the main features of both Petri net types. FMC-PNDs are better suited for stakeholders. The idea of translating UML 2 activity diagrams into FMC-PND simplifies many of the difficulties encountered in transforming UML activities into other classes of Petri nets. From experience, basic patterns are useful in over $90 \%$ of all scenarios. FMCPND cover other types of patterns that can be extended to this work e.g. producer/consumer, recursion, inter task, etc.

\section{Conclusion}

This topic has dealt with UML activities, FMC-PND and CPNs. There is evidence suggesting intuitive, informal or formal mapping of UML activities into their Petri net counterparts. The models or examples given in the UML super structure specification are all transformable into CPNs. Certain classes of UML activities follow closely the behavior of Petri nets. This topic is supported with a vast amount of literature. UML 2 activities are 
based on Petri net semantics. They can be suitably represented using reduced FMC-PND which are more comprehensible than a complete CPN. The FMC-PND has to be converted into a CPN for more detailed verification and simulation. CPNs are best suited for detailed modelling and execution of activity diagrams. Other constructs like pins, complex nodes, hierarchy can also be included. CPN modeling is suitable for i) fundamental, ii) basic and iii) intermediate activities of UML, although this can be extended to other classes. Activity diagrams and their semantics are important for model driven approaches. Model driven approaches used for requirements engineering, offer better choices than code driven approaches. When modelling business processes, workflows, complex software and information systems, model driven approaches are preferable. The FMC-PNDs are suitable for representing repeated patterns in activity models using a concise notation that is simpler to comprehend and visualize.

CPN and higher order net semantics are richer and more expressive than activity semantics. This implies that even if direct one-to-one mapping is done using TGG (triple graph grammars) or the OMG QVT, many additional details like type definitions, token types, place types, firing rules, functions for transitions and arcs, etc. must be added to make the CPN a proper detailed executable model. Functions, in particular, require programming using the ML language. CPN's seem to be the best natural choice for modeling FMC-PNDs and UML activities. The CPN is suitable for detailed stepwise execution and optimization. If the CPN is mapped to the activity using TGG correspondence any change to the CPN's structure will automatically update the activity model accordingly. Petri nets and Colored Petri nets are translatable into formal languages and can be expressed using other notations. Activity diagrams can be formalized to a certain extent using the UML's OCL (object constraint language).

As previously explained, the approaches to transform activities into Petri net models can be i) formal or ii) informal. Different classes of Petri nets can be used for different needs. To capture all the detail from the activity model, CPNs are definitely the best choice. However on some occasions a simple place transition Petri net could have its valid use. Petri nets offer us visual formalisms useful for examining system properties. A reduced Petri net like the FMC-PND is suitable for better comprehension.

\section{References}

Baresi, L. \& Pezze, M. (2001). Improving UML with Petri Nets, Electronic notes in Theoretical Computer Science, pp. 107-119, UNIGRA, doi: 10.1016/ S1571-(04)80947-2 Vol 44., No. 2, Jul 2007, Elsevier

Bock, C. (2005). SysML and UML 2 support for Activity Modeling, International Journal Council of Systems Engineering, Vol. 9 No. 2, Nov 2005, pp. 160-186, WileyInterScience DOI 10.1002/sys.20046 www.interscience.wiley.com

Borger, E.; Cavara, A. \& Riccobene, E. (2000). An ASM Semantics for UML Activity Diagrams, Proc. of $8^{\text {th }}$ International Conference on Algebraic Methodology and Software Technology, pp. 293 - 308, ISBN: 3-540-67530-2, Iowa City, May 2000, SpringerVerlag, UK

Canevet, C.; Gilmore, S. , Hilliston, J., Kloul, L. \& Stevens, P. (2004). Analysing UML 2.0 Activity Diagrams in the Software Engineering Performance Process, Proc. of 
WOSP'04,pp. 77-78, ISBN ISSN: 0163-5948,1-58113-673-0, Redwood City CA, Jan 2004, ACM

Chen, S.; Eshaghian, M. \& Wu, Y.C. (1995). Mapping Arbitrary Non-Uniform Task Graphs onto Arbitrary Non-Uniform System Graphs, Proceedings of the 1995 International Conference on Parallel Processing, pp. 191-195, ISBN: 0-8493-2616-8, UrbanaChampain, Illinois, USA, Aug 1995, CRC Press, USA

CPNTools (2009). CPN Group, Department of Computer Science, University of Aarhus, Denmark http://www.daimi.au.dk/CPnets/

Dori, D. (2003). Conceptual Modeling and System Architecture, Communications of the ACM, Vol 46 No 10, Oct 2003, pp. 62-65, ISSN: 0001-0782

Dumas, M. \& Ter Hofstead, A.H.M. (2001). UML Activity Diagrams as a Workflow Specification Language, Proceedings of the 4th International Conference on The Unified Modeling Language, Modeling Languages, Concepts, and Tools, pp. 76-90, ISBN: 978-3540-42667-7, Toronto, Oct 2001, Springer-Verlag, Berlin

Garrido, J.L. \& Gea, M. (2002). A Colored Petri Net Formalization for a UML-based Notation Applied to Cooperative System Modeling, Interactive Systems: Design, Specification and Verification, LNCS 2545, pp.16-28, Springer-Verlag, ISBN: 978-3540-00266-6, Berlin/Hiedelberg

Ghodsi, M. \& Kent, K. (1991). Well-formed Generalized Task Graphs, Proceedings of the 3rd IEEE Symposium on Parallel and Distributed Processing, pp.344-351, ISBN: 0-81862310-1, Dallas TX, Feb 1991, IEEE, USA

Gröne, B. \& Tabeling, P. (2003). A System of Conceptual Architecture Patterns for Concurrent Request Processing Servers, Proc. of 2nd Nordic Conference on Pattern Languages of Programs VikingPLOP03, Bergen, Norway, Oct 2003

Gröne, B.; Knöpfel, A., Kugel, R. \& Schmidt, O. (2004). The Apache Modeling Project, Technical Report 5, Hasso-Plattner-Institute, Potsdam, 2004. http:/ / www.f-m-c.org

Gröne, B. (2006). Conceptual Patterns, Proc. of $13^{\text {th }}$ IEEE International Symposium and Workshop on Engineering of Computer Based Systems, pp. 241-246, ISBN: 0-7695-25466, ECBS, Potsdam, Germany, Mar 2006, IEEE,USA

Hu, Z. \& Shatz, S.M. (2004). Mapping UML Diagrams into a Petri Net Notation for System Simulation, Proceedings of the Sixteenth International Conference on Software Engineering \& Knowledge Engineering (SEKE'2004),pp, 213-219, ISBN: 1-891706-14-4, Illinios Chicago, Jun 2004, Knowledge Systems Institute Grad. School, Banff, Alberta, Canada

Jancăr, P.; Esparza, J. \& Moller F. (1999). Petri Nets \& Regular Processes, Journal of Computer and System Sciences, Vol. 59, No. 3, Dec 1999, pp. 476-503, ISSN: 0022-0000

Jørgensen, J.B. (2002). Coloured Petri Nets in UML-Based Software Development Designing Middleware for Pervasive Healthcare, Proc. $4^{\text {th }}$ Int. Workshop on the Practical use of CPN \& CPN Tools Aug 2002, pp. 61-80, Diami Technical Report PB560, Aarhus Denmark

Jørgensen, J.B. \& Lassen, K.B. (2006). Requirements Engineering for the Adviser Portal Bank System, Proc. of 13th IEEE International Symposium and Workshop on Engineering of Computer Based Systems ECBS, pp. 259-268, ISBN: 0-7695-2125-8, Potsdam, Germany, Mar 2006,IEEE, N.Y.

Kaindl, J.H. \& Carroll, J.M. (1999). Symbolic Modeling in Practice, Communications of the ACM, Vol. 42 No. 1, Jan 1999, pp. 28-30, ISSN: 0001-0782 
Kindler, E. \& Wagner, R. (2007). Triple Graph Grammers: Concepts, Extensions, Implementations and Application Scenarios, Technical Report Tr-ri-284, University of Paderborn, Paderborn, Germany, 2007

King, P. \& Pooley, R. (2002). Derivation of Petri Net Models from UML Specifications of Communication Software, Proc. of 11th Int. Conf. On Tools and Techniques for Computer Performance Eval, pp. 262-276, ISBN: 3-540-67260-5, Illinois USA, Mar 2002, Springer-Verlag, London, UK

Knöpfel, A.; Gröne, B. \& Tabeling, P. (2006). Fundamental Modeling Concepts, Wiley, ISBN13:978-0-470-02710-3, West Sussex, UK

Kristensen, L.M.; Christensen, S. \& Jensen, K. (1998). The Practioner's Guide to Coloured Petri Nets, International Journal On Software Tools for Tech. Transfer (STTT), Vol. 2, No.2, Dec 1998, pp. 98-132, ISSN: 1433-2779, 1433-2787

Kristensen, L.M.; Jørgensen, J.B. \& Jensen K. (2004). Application of Coloured Petri Nets in System Development, Lecture Notes in Computer Science, 2004, Vol. 3098/2004, pp. 99-27, ISSN: 0302-9743, 1611-3349

LaQuSo (2007). LaQuSo Work Group / Project, LaQuSo Repository, Eindhoven, www.Laquso.com

Lohmann, C., Greenyer, J. , Jiang, J. \& Systä, T. (2007) Applying Triple Graph Grammars For Pattern-Based Workflow Model Transformations, Journal of Object Technology, Special Issue:Tools, pp. 253-273, Oct 2007, Europe,

http://www.jot.fm/issues/issue_2007_10/paper13/

Lopez-Grao, J.P. \& Campos J. (2004). From UML Activity Diagrams to Stochastic Petri Nets: Application to Software Performance Engineering, ACM Sigsoft Software Engineering Notes, Vol. 29, No. 3, -36, 2004, pp. 25-36, ISSN: 0163-5948

Lorenz, A. \& Six, H.W. (2006). Tailoring UML Activities to Use Case Modeling for Web Application Development, Proceedings of the conference of the Centre for Advanced Studies on Collaborative Research, pp. 333-338, Toronto, Oct 2006, IBM, Toronto

Merseguer, J.; Campos, J \& Mena, E. (2006). On the Integration of UML and Petri Nets in Software Development, Proc. of ICATPN'06 LNCS 4024, pp.19-36, ISSN: 0302-9743, Turku Finland, Jun 2006, Springer, Berlin

OMG (2009).UML2 Superstructure Specification. V2.2, OMG, http://www.omg.org/technology/documents/formal/uml.htm

Petriu, D.C. \& Shen, H. (2002). Applying the UML Performance Profile: Graph Grammer based derivation of LQN models from UML Specifications, Proceedings of the $12^{\text {th }}$ International Conference on Computer Performance Evaluation Modelling Techniques and Tools, pp. 159-177, ISBN: 3-540-43539-5, London, 2002, Springer-Verlag, UK

Saldhana, J.A. \& Shatz, S.M. (2000). UML Diagrams to Object Petri Net Models: An Approach for Modeling \& Analysis, SEKE 2000, pp. 103-110, ISBN: 1891706955/9781891706059, Chicago Illinois, Jul 2000, Knowledge Systems Institute, USA

Shin, M.E.; Levis, A.H. \& Wangenhals, L.W. (2003). Transformation of UML-Based System Model to Design/CPN model for Validating System Behavior, Proc. of the 6th Int. Conf. on the UML/Workshop on Compositional Verification of the UML Models, pp. 3-22, doi: 10.1016/j.entcs.2005.12.059, Electronic Notes in Theoretical Computer Science, Vol. 159, May 2006, Elsevier Science. 
Shinkawa, Y. (2006). Inter-Model Consistency in UML based on CPN Formalism, Proc. of APSEC'06, pp. 411-418, ISSN: 1530- 1362, 0- 7695-2685-3, Bangalore India, Dec 2006, IEEE, USA

Sivaraman, E. \& Kamath M. (2003). On the use of Petri Nets for Business Process Modeling, On The Move to Meaningful Internet Systems 2003: OTM 2003Workshops, Business Contract Obligation Monitoring through Use of Multi Tier Contract Ontology LNCS 2889/2003, Oct 2003, pp. 690-702, ISSN: 0302-9743

Soderborg, N.R.; Crawley, E.F. \& Dori, D. (2003). OPM- Based Definitions \& Operational Templates, Communications of the ACM, Vol. 46 No. 10, Oct 2003, pp. 67-72, ISSN:0001-782

Spiteri Staines, T. (2008). Intuitive Mapping of UML 2 Activity Diagrams into Fundamental Modeling Concept Petri Net Diagrams and Colored Petri Nets, Proc. of the $15^{\text {th }}$ IEEE International Symposium and Workshop on Engineering of Computer Based Systems, pp. 191-200, ISBN: 0-7695-3141-5, Belfast, Ireland, ECBS, Apr 2008, IEEE, USA

Störrle, H. (2004a). Structured Nodes in UML 2.0 Activities, Nordic Journal of Computing, Vol. 11, No. 3, Sep 2004, pp. 279-302, ISSN: 1236-6064

Störrle, H. (2004b). Semantics of Control Flow in UML 2.0 Activities, Proc. of 2004 IEEE Symposium on Visual Languages and Human Centric Computing, pp. 235-242, ISBN: 0-7803-8696-5, 2004,IEEE, USA

Störrle, H. \& Hausmann, J.H. (2005). Reasoning about UML Activity Diagrams, Publ. Assoc. Nordic Journal of Computing, Vol. 14 No. 1, 2005, pp.43-64, ISSN: 1236-6064

Tabeling, P. \& Gröne, B. (2005). Integrative Architectural Elicitation for Large Scale Computer Based Systems, Proc. of the 12th IEEE International Symposium and Workshop on Engineering of Computer Based Systems, pp. 51-61, ISBN: 0-7695-2308-0, Green Belt, MD, ECBS, Apr 2005, IEEE, USA

Tabeling, P. (2002). Multi Level Modeling of Concurrent and Distributed Systems, Proc. of the International Conference on Software Engineering Research and Practice. CSREA Press, Jun 2002, http://www.fmc-modeling.org/download/publications/tabeling_2002multi-level_modeling_of_concurrent_and_distributed_systems.pdf

Tabeling, P. (2004). Architectural Description with Integrated Data Consistency Models, Proc. of the 11 th IEEE International Symposium and Workshop on Engineering of Computer Based Systems, pp. 178- 185, ISBN: 0-7695-2125-8, Brno Czeck Republic, ECBS, May 2004, IEEE, USA

Van der Aalst, W.M.P. (1998). The Application of Petri Nets to Workflow Management. Lecture Notes, Eindhoven University of Technology, Eindhoven, http:/ / tmitwww.tm.tue.nl/staff/wvdaalst/publications/p53.pdf

Van Hee, K.M. (1995). Information Systems Engineering A Formal Approach, Cambridge University Press, ISBN: 0-521-45514-6, N.Y. USA

Van Hee, K.M. (2005). Information Systems Architecture A Practical and Mathematical Approach, Lecture Notes, Technische Universiteit Eindhoven, 2005, http://wwwis.win.tue.nl/ wsinhee/sm1/

Ziaei, R. \& Agha, G.(2003). SynchNet : A Petri Net Based Coordination Language for Distributed Objects, Generative Programming and Component Engineering LNCS 2830/2003, Springer Verlag, Vol 48, 2003, pp. 324-343, ISBN: 3-540-20102-5, N.Y. 


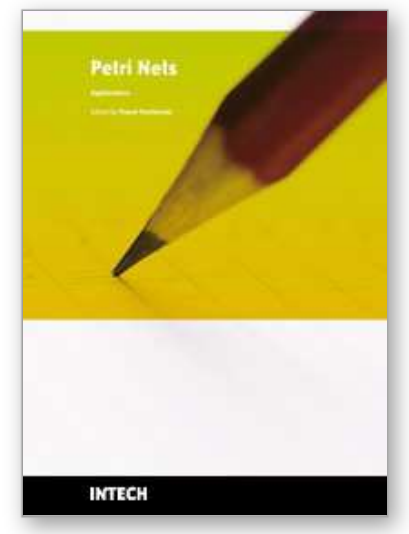

\author{
Petri Nets Applications \\ Edited by Pawel Pawlewski
}

ISBN 978-953-307-047-6

Hard cover, 752 pages

Publisher InTech

Published online 01, February, 2010

Published in print edition February, 2010

Petri Nets are graphical and mathematical tool used in many different science domains. Their characteristic features are the intuitive graphical modeling language and advanced formal analysis method. The concurrence of performed actions is the natural phenomenon due to which Petri Nets are perceived as mathematical tool for modeling concurrent systems. The nets whose model was extended with the time model can be applied in modeling real-time systems. Petri Nets were introduced in the doctoral dissertation by K.A. Petri, titled “"Kommunikation mit Automaten” and published in 1962 by University of Bonn. During more than 40 years of development of this theory, many different classes were formed and the scope of applications was extended. Depending on particular needs, the net definition was changed and adjusted to the considered problem. The unusual "flexibility" of this theory makes it possible to introduce all these modifications. Owing to varied currently known net classes, it is relatively easy to find a proper class for the specific application. The present monograph shows the whole spectrum of Petri Nets applications, from classic applications (to which the theory is specially dedicated) like computer science and control systems, through fault diagnosis, manufacturing, power systems, traffic systems, transport and down to Web applications. At the same time, the publication describes the diversity of investigations performed with use of Petri Nets in science centers all over the world.

\title{
How to reference
}

In order to correctly reference this scholarly work, feel free to copy and paste the following:

Anthony Spiteri Staines (2010). Intuitive Transformation of UML2 Activities into Fundamental Modeling Concept Petri Nets and Colored Petri Nets, Petri Nets Applications, Pawel Pawlewski (Ed.), ISBN: 978-953307-047-6, InTech, Available from: http://www.intechopen.com/books/petri-nets-applications/intuitivetransformation-of-uml2-activities-into-fundamental-modeling-concept-petri-nets-and-colored

\section{INTECH}

open science | open minds

\section{InTech Europe}

University Campus STeP Ri

Slavka Krautzeka 83/A

51000 Rijeka, Croatia

Phone: +385 (51) 770447

Fax: +385 (51) 686166

www.intechopen.com

\section{InTech China}

Unit 405, Office Block, Hotel Equatorial Shanghai

No.65, Yan An Road (West), Shanghai, 200040, China 中国上海市延安西路65号上海国际贵都大饭店办公楼405单元

Phone: +86-21-62489820

Fax: +86-21-62489821 
(C) 2010 The Author(s). Licensee IntechOpen. This chapter is distributed under the terms of the Creative Commons Attribution-NonCommercial-ShareAlike-3.0 License, which permits use, distribution and reproduction for non-commercial purposes, provided the original is properly cited and derivative works building on this content are distributed under the same license. 